\title{
Error Exponents for the Detection of Gauss-Markov Signals Using Randomly Spaced Sensors
}

\author{
Saswat Misra, Member, IEEE, and Lang Tong, Fellow, IEEE
}

\begin{abstract}
We derive the Neyman-Pearson error exponent for the detection of Gauss-Markov signals using randomly spaced sensors. We assume that the sensor spacings, $d_{1}, d_{2}, \ldots$, are drawn independently from a common density $f_{d}($.$) , and we$ treat both stationary and nonstationary Markov models. Error exponents are evaluated using specialized forms of the Strong Law of Large Numbers, and are seen to take on algebraically simple forms involving the parameters of the Markov processes and expectations over $f_{d}($.$) of certain functions of d_{1}$. These expressions are evaluated explicitly when $f_{d}($.$) corresponds to$ i) exponentially distributed sensors with placement density $\lambda$; ii) equally spaced sensors; and iii) the proceeding cases when sensors fail (or equivalently, are asleep) with probability $q$. Many insights follow. For example, we determine the optimal $\lambda$ as a function of $q$ in the nonstationary case. Numerical simulations show that the error exponent predicts trends of the simulated error rate accurately even for small data sizes.
\end{abstract}

Index Terms-Error exponent, Gauss-Markov, NeymanPearson detection, optimal placement density, sensors.

\section{INTRODUCTION}

W E study the detection of a correlated signal field by a set of sensors and a fusion center (FC), as depicted in Fig. 1. We assume that the signal is present under both hypotheses, $\mathcal{H}_{j}, j \in\{0,1\}$, and that it has a Gauss-Markov correlation structure and power level that are hypothesis-dependent. The signal field is sampled by $N$ sensors, and these samples are collected by the FC. The FC then makes a global decision as to the true hypothesis using Neyman-Pearson (NP) hypothesis testing [21]. We assume that the sensors are randomly located along a straight line. The assumption that sensors are on a straight line models scenarios such as when the fusion center is a mobile collection agent (e.g., a unmanned rover) that traverses the network to collect data, and/or when the sensor network is in the far field of the signal source [19]. The assumption of randomness models the fact that sensors are often deployed without precise control (e.g., they are air dropped in military applications). Even if sensors are deterministically spaced upon deployment, mechanical failures and/or sleep cycles introduce randomness into the spacing between the operational sensors.

Manuscript received March 20, 2007; revised January 7, 2008. The associate editor coordinating the review of this manuscript and approving it for publication was Dr. Cedric Richard.

S. Misra is with the Army Research Laboratory, Adelphi, MD 20783 USA (e-mail: sm353@cornell.edu).

L. Tong is with the School of Electrical and Computer Engineering, Cornell University, Ithaca, NY 14853 USA (e-mail: Itong@ece.cornell.edu).

Color versions of one or more of the figures in this paper are available online at http://ieeexplore.ieee.org.

Digital Object Identifier 10.1109/TSP.2008.919106

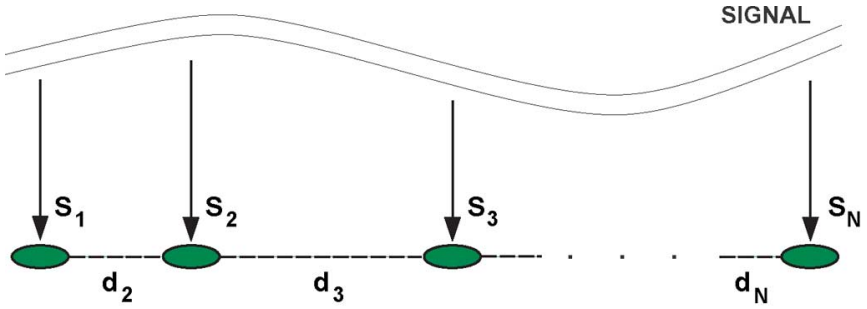

FUSION CENTER

Fig. 1. The collection of $N$ signal samples from randomly placed sensors $\left\{s_{k}\right\}_{k=1}^{N}$ denotes the collected samples, and $\left\{d_{k}\right\}_{k=2}^{N}$ denotes the (random) sensor spacings. The data $\left\{s_{k}\right\}$ and $\left\{d_{k}\right\}$ are collected by a FC, which makes a global decision as to the true hypothesis using the Neyman-Pearson framework.

We study the theoretical detection performance once the $N$ samples arrive at the FC. ${ }^{1}$

As an example in which this model is relevant, consider sensors deployed ad-hoc in a hostile environment and tasked with classifying a passing tank as either friendly (hypothesis $\mathcal{H}_{0}$ ) or enemy $\left(\mathcal{H}_{1}\right)$, based on the acoustic wavefront that the tank produces. This wavefront is a signal field that can be sampled by acoustic sensors. The power and correlation structure of these samples would depend on the tank's class as well as the (random) locations of sensors.

\section{A. Background}

Consider a general binary hypothesis test between $\mathcal{H}_{0}$ and $\mathcal{H}_{1}$. Let $\mathbf{y}=\left[y_{1}, \ldots, y_{N}\right]^{T} \in \mathbb{R}^{N}$ be a vector of observed data, and assume that the NP framework is used. Let $P_{F}(N)$ and $P_{M}(N)$ denote the probability of false alarm and probability of miss as functions of $N$, respectively. The NP error exponent is defined, for a fixed constraint $P_{F}(N) \leq \alpha \in(0,1)$, as the exponential rate of decay in $P_{M}(N)$ as the number of data samples approaches infinity, i.e.,

$$
K \triangleq \lim _{N \rightarrow \infty}-\frac{1}{N} \ln P_{M}(N)
$$

provided that the limit exists. $K$ is a useful metric. It provides an estimate on the number of observations needed to attain a given level of detection performance, and is often parameterized by physical and design parameters [e.g., the signal-to-noise ratio (SNR) and sensor spacing] that can be optimized to improve the

\footnotetext{
${ }^{1}$ The communication protocols used to initiate the detection process and to deliver the samples to the FC are not considered in this work.
} 
detection performance. However, $K$ is currently in an implicit form that is not amenable to analysis.

Let $p_{j}(\mathbf{y})$ denote the probability density of $\mathbf{y}$ when $\mathcal{H}_{j}$ is true. If the likelihood ratio test (LRT) is used at the FC, it can be shown through a generalization of Stein's lemma that [27]

$$
K=\lim _{N \rightarrow \infty} \frac{1}{N} \ln \frac{p_{0}(\mathbf{y})}{p_{1}(\mathbf{y})} \quad\left(\text { a.s. in } \mathcal{H}_{0}\right)
$$

provided that the limit exists, where the notation (a.s. in $\mathcal{H}_{0}$ ) means that the limit is to be taken in the almost sure sense under $\mathcal{H}_{0}$. Note that (2) is independent of $\alpha$.

\section{B. Related Work}

For a general overview of multisensor detection, see [28]. For a discussion of issues related to multisensor detection with correlated sensor observations, see [1], [4], [7], [9], [16], and the references therein. This paper addresses the multisensor detection of correlated signals using randomly placed sensors and the NP error exponent (1).

Most related to this paper are [24], [25], [3, pp. 138-139], [18], and [19], each of which considers correlated signals and evaluates the criterion (1) for deterministically spaced sensors. In [24], the authors derive the error exponent when the signal is a stationary Gauss-Markov signal under one hypothesis, and independent and identically distributed (i.i.d.) noise under the other. In [25], this work is extended to analyze the optimal arrangement of sensors along a spatial line. The error exponent is found using implicit solutions of certain matrix equations. An extensive numerical analysis is used to characterize the optimal sensor spacing as a function of the SNR and field correlation. It is observed that either a uniform or a clustered approach to sensor spacing appears to be optimal, depending on the field correlation and SNR. When clustering is optimal, there appears to be an optimal cluster size. In [3, pp. 138-139] and [18], error exponents are derived assuming Gauss-Markov signals under both hypotheses. In [19], we previously derived properties of the error exponent for Gauss-Markov signals under both hypotheses, using a physical model which linked the correlation parameter to network design parameters.

In the Bayesian setup, error exponents based on the Chernoff information or the similar Bhattacharya bound can be found in [5], [6], [15], and [23], among others. In [15], the Chernoff and Bhattacharya bounds are used to determine the optimal number of sensors for communications to a FC under power and bandwidth constraints. In [5] and [6], the error exponent is derived when sensors have dependent observations. The optimal sensor density is studied when sensors are equally spaced, using both numerical techniques and closed-form expressions (depending on the assumptions). In [23], a routing scheme is designed based on the Chernoff information for a deterministic sensor placement.

However, none of these works provide insights for the case when sensors are randomly located, motivating the need for the analysis presented in this paper.

\section{Organization and Main Results}

In Sections II and III, we derive $K$ for Gauss-Markov signal models assuming that sensor spacings $d_{1}, d_{2}, \ldots$, are drawn i.i.d. from an arbitrary density function $f_{d}($.$) . Error exponents$ are seen to take on algebraically simple forms involving the parameters of the Markov processes and expectations over $f_{d}($.$) of certain functions of d_{1}$. In Section II, we consider the nonstationary case. We show that the error exponent simplifies to closed form expressions under the following (physically motivated) special cases of $f_{d}($.$) : (i) exponentially distributed$ sensors with placement density $\lambda$; (ii) equally spaced sensors with spacing $\bar{d}$, and (iii) the proceeding cases when sensors fail with probability $q$. For exponentially distributed sensors with failures, the optimal sensor placement density is found in closed form. In Section III, we consider the stationary case. We evaluate the error exponent in closed form, in terms of the Psi function, when $f_{d}($.$) corresponds to exponentially distributed$ sensors with failures. This expression is seen to simplify in the limit of sparsely and densely placed sensors. Numerical simulations are used throughout to show that the error exponent predicts trends of the simulated error rate accurately, for even small data sizes. Thus, the analytic framework presented here allows for an accurate and efficient optimization of system resources that would not be possible otherwise. Finally, as a matter of organization style, we defer all proofs to the Appendices.

\section{Notation}

We use the following additional notation and definitions: (a) $\mathbb{E}[$.$] denotes expectation. When there is potential ambiguity,$ $\mathbb{E}_{X}[$.$] denotes expectation with respect to a random variable X$, and $\mathbb{E}_{\mathcal{H}_{j}}[$.$] denotes expectation with respect to the hypothesis$ $\mathcal{H}_{j}$, (b) $X \sim f_{X}(x)$ denotes that the random variable $X$ is distributed according to the density function $f_{X}(),.(c) \mathcal{N}\left(0, \sigma^{2}\right)$ denotes a zero mean Gaussian random variable with variance $\sigma^{2},(\mathrm{~d})$ if $f(x)=O\left(x^{k}\right)$, then $|f(x)|<C x^{k}$ for some $C \in \mathbb{R}^{+}$ and all sufficiently small $x$, and (e) boldface lowercase letters, e.g., $\mathbf{x}$, denote vectors.

\section{NONSTATIONARY GAUSS-MARKOV MODEL}

Nonstationary Gauss-Markov models have been used to describe communications signals in a wide variety of contexts (e.g., see [12]-[14], and the references therein). Here, we model the signal under each hypothesis as a Gaussian signal that evolves with a Markov correlation structure along any straight line. Consider the observations $\left\{s_{k}\right\}_{k=1}^{N}$ taken by the sensors. We assume that the statistics of $\left\{s_{k}\right\}$ under $\mathcal{H}_{j}$ are described by

$$
\begin{aligned}
& s_{1} \sim \mathcal{N}\left(0, \sigma_{j, I}^{2}\right) \\
& s_{k}=a_{j, k} s_{k-1}+z_{j, k}, \quad k \geq 2
\end{aligned}
$$

where $a_{j, k}$ describes the correlation strength between the ( $k-$ 1 )th and $k$ th sensors, and $z_{j, k} \stackrel{i . i . d .}{\sim} \mathcal{N}\left(0, \sigma_{j}^{2}\right)$ is impulsive (or innovations) noise. We assume that $\sigma_{j, I}^{2}, \sigma_{j}^{2} \in(0, \infty)$ for $j \in$ $\{0,1\}$. Let $\left\{d_{k}\right\}_{k=2}^{N}$ be the i.i.d. sequence of sensor spacings. We assume that $d_{k} \sim f_{d}(x)$, where $f_{d}(x), x \geq 0$, is either a continuous or discrete probability density function which is independent of $\mathcal{H}_{j}$, and that $a_{j, k}$ is a function of $d_{k}$, i.e.

$$
a_{j, k}=g_{j}\left(d_{k}\right)
$$


where $g_{j}(x) \in(0,1)$ is a hypothesis-dependent deterministic function (for an example, see (5)). We assume that $\left\{s_{k}\right\}_{k=1}^{N}$ and $\left\{d_{k}\right\}_{k=2}^{N}$ are obtained by the FC. ${ }^{2}$ Finally, it will be convenient to define $d \triangleq d_{1}$ and $a_{j} \triangleq g_{j}\left(d_{1}\right)$ for use in expressions where the index $k$ is irrelevant.

Usually in sensor array processing applications, the data received across sensors is a function of: (a) the signal emitted by the source, (b) the propagation characteristics of the environment, (c) the sensor locations, and (d) the signal direction of arrival. In the model above, $g_{j}(x), \sigma_{j, I}^{2}$, and $\sigma_{j}^{2}$ capture the effects of (a)-(c) under $\mathcal{H}_{j}$ (illustrative examples will be given in the sequel). Although we do not specifically incorporate (d) into our model, an example of how to do so is given in [19].

\section{A. Derivation of the Error Exponent}

Notice that $\mathbf{y}=[\mathbf{d}, \mathbf{s}]$ summarizes the data obtained by the FC, where $\mathbf{d} \triangleq\left[d_{2}, \ldots, d_{N}\right]$ and $\mathbf{s} \triangleq\left[s_{1}, \ldots, s_{N}\right]$. Evaluating the log-likelihood ratio in (2), we get

$$
\begin{aligned}
& \ln \frac{p_{0}(\mathbf{s}, \mathbf{d})}{p_{1}(\mathbf{s}, \mathbf{d})} \stackrel{(a)}{=} \ln \frac{p_{0}(\mathbf{s} \mid \mathbf{d})}{p_{1}(\mathbf{s} \mid \mathbf{d})} \stackrel{(b)}{=} \ln \frac{p_{0}\left(s_{1}\right)}{p_{1}\left(s_{1}\right)} \prod_{k=2}^{N} \frac{p_{0}\left(s_{k} \mid s_{k-1}, d_{k}\right)}{p_{1}\left(s_{k} \mid s_{k-1}, d_{k}\right)} \\
& \stackrel{(c)}{=} \frac{1}{2} \ln \frac{\sigma_{1, I}^{2}}{\sigma_{0, I}^{2}}+s_{1}^{2} \frac{\sigma_{0, I}^{2}-\sigma_{1, I}^{2}}{2 \sigma_{0, I}^{2} \sigma_{1, I}^{2}}+\frac{N-1}{2} \ln \frac{\sigma_{1}^{2}}{\sigma_{0}^{2}} \\
& +\sum_{k=2}^{N}\left\{\frac{\left(s_{k}-a_{1, k} s_{k-1}\right)^{2}}{2 \sigma_{1}^{2}}\right. \\
& \left.-\frac{\left(s_{k}-a_{0, k} s_{k-1}\right)^{2}}{2 \sigma_{0}^{2}}\right\}
\end{aligned}
$$

where $(a)$ follows since $p_{j}(\mathbf{d})$ is independent of $j,(b)$ follows since $\mathbf{s}$ is a Markov process given $\mathbf{d}$, and $(c)$ follows from the form of the conditional Gaussian distribution. In Appendix I, we reference three useful forms of the Strong Law of Large Numbers (SLLN). Using these results, we take the almost sure limit of (3) in Appendix II. We arrive at the following theorem.

Theorem 2.1: Suppose $\mathbb{E}_{d}\left[g_{0}(d)^{2}\right]<1$. Then the NP error exponent for the detection of nonstationary Gauss-Markov signals with randomly placed sensors is

$$
K=\frac{1}{2}\left\{\ln R-1+\frac{1}{R}\left(1+\frac{\mathbb{E}_{d}\left[\left(g_{0}(d)-g_{1}(d)\right)^{2}\right]}{1-\mathbb{E}_{d}\left[g_{0}(d)^{2}\right]}\right)\right\}
$$

where $R \triangleq \sigma_{1}^{2} / \sigma_{0}^{2}$.

The proof relies on bounding the correlation function of certain sequences appearing in (3), so as to apply SLLN-3 (Appendix I), which is applicable to certain nonstationary processes. We emphasize that $f_{d}($.$) can be either a continuous$ or discrete density (examples of each are given in Section II-B). If the sensor spacing is deterministic, the expectations above disappear, and it can be verified that (4) reduces to the error exponent given previously in [18], as it must.

\footnotetext{
${ }^{2}$ If the FC is a mobile collection agent, it can measure $\left\{d_{k}\right\}$ directly. Other-
} wise, sensors can send $\left\{d_{k}\right\}$ to the FC, if they are equipped with GPS.

\section{B. Example}

We evaluate (4) explicitly for two models of the sensor spacing. In each, we assume that $a_{j, k}$ decays exponentially in $d_{k}$ at a rate proportional to a constant $A_{j}$, i.e.,

$$
g_{j}(x)=e^{-A_{j} x}
$$

for $x \geq 0$, where $A_{j} \in(0, \infty)$ is known at the $\mathrm{FC}$, and where $A_{0} \neq A_{1}$. We assume that sensors are in "failure" with probability $q \in[0,1)$ independently from sensor to sensor. Specifically, sensor $k$ is said to be in failure if its data $\left(s_{k}, d_{k}\right)$ is not received at the FC. Typical reasons for failure include mechanical malfunction or battery depletion at the sensor, and lost transmissions due to interference at the FC. In addition, by choosing $q$ appropriately, the analysis below incorporates probabilistic transmission schemes (in which a node transmits its data only with some probability; such a scheme was shown to provide the optimal tradeoff between error exponents and energy consumption in [26]) and schemes in which sensors enter cycled "sleep" states.

1) Exponentially Spaced Sensors With Failures: Let the sensor spacings be exponentially distributed with placement density (or "arrival rate") $\lambda$ and failure rate $q$. We have

$$
f_{d}(x)=\lambda(1-q) e^{-\lambda(1-q) x}, \quad x \geq 0
$$

where $\lambda=1 / \mathbb{E}[d]$, and where (6) is valid for $q>0$ by the "thinning" property of the Poisson process [10, p. 287]. ${ }^{3}$ Evaluating (4) with (5) and (6), we get

$$
K=\frac{1}{2}\left\{\ln R-1+\frac{1}{R}\left(1+\frac{\left(A_{1}-A_{0}\right)^{2} \lambda_{q}}{A_{0}\left(A_{0}+A_{1}+\lambda_{q}\right)\left(2 A_{1}+\lambda_{q}\right)}\right)\right\}
$$

where $\lambda_{q} \triangleq \lambda(1-q)$. The proof follows from algebraic manipulation and is omitted for brevity.

It may be suspected that there exists a maximizing $\lambda \in(0, \infty)$ in (7). To understand why, fix $N<\infty$. As $\lambda \rightarrow \infty$, the $N$ sensors will be placed arbitrarily close together, and their observations $\left\{s_{k}\right\}_{k=1}^{N}$ will cover an arbitrarily small evolution of the (random) signal. Conversely, as $\lambda \rightarrow 0$, the $N$ sensors will be placed arbitrarily far apart, and their observations will approach an i.i.d. set. Thus, the correlation in the signal field cannot be exploited in the detection process. Indeed, it can be verified that (7) is unimodal in $\lambda$ and is maximized when

$$
\lambda=\lambda^{\star} \triangleq \frac{\sqrt{2 A_{1}\left(A_{0}+A_{1}\right)}}{1-q}
$$

i.e., there exists an optimal placement density.

Assume that time and energy are required for sensors to transmit their data to the FC. It then makes sense for the FC to limit the number of sensors that are polled during each "detection process". Given that $N$ sensors are to be polled, (8) provides guidance, increasingly accurately as $N$ increases, on which sensors should be polled.

\footnotetext{
${ }^{3}$ More formally, we have that $d_{k}=\sum_{\ell=1}^{M} \tilde{d}_{k, \ell}$, where $\left\{\tilde{d}_{k, \ell}\right\}_{\ell}$ is an i.i.d. sequence with common probability density given by the right-hand side (RHS) of (6) with $q=0$, and where $P[M=k]=q^{k-1}(1-q)$ for $k \in\{1,2, \ldots\}$. It can be verified that the probability density of $d_{k}$ is $f_{d}(x)=\lambda(1-q) e^{-\lambda(1-q) x}$.
} 


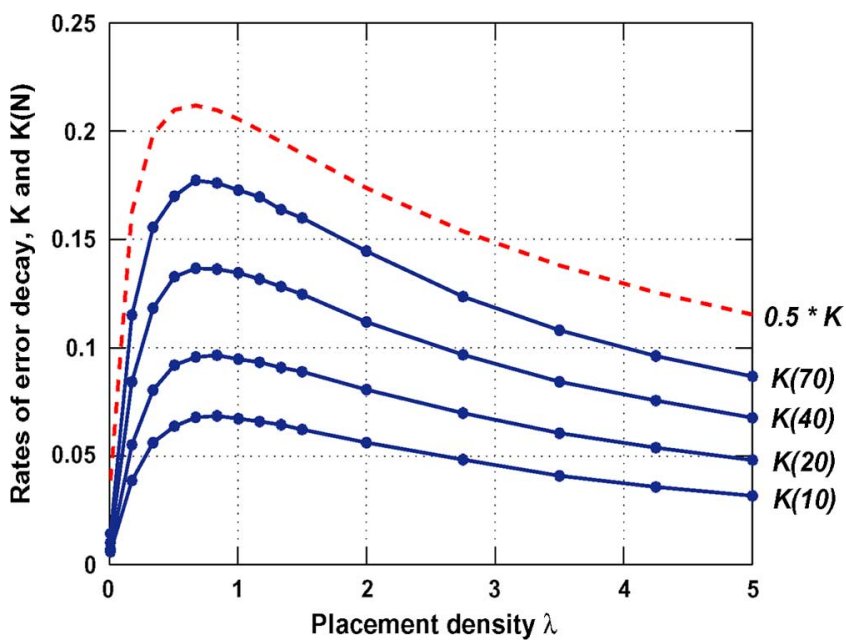

Fig. 2. NP detection performance versus placement density for exponentially distributed sensors. Plot of $K$ and $K(N)$ (for $N \in\{10,20,40,70\}$ ) versus $\lambda$ for $A_{0}=2, A_{1}=0.1, \sigma_{0}^{2}=3 / 2, \sigma_{1}^{2}=1, \sigma_{0, I}^{2}=\sigma_{1, I}^{2}=0.5, q=0$, and $\alpha=0.01$.

To study the usefulness of $K$ in predicting the behavior of the error rate for finite $N$, we use numerical simulations. Define the finite- $N$ exponential error rate to be

$$
K(N) \triangleq-\frac{1}{N} \ln P_{M}(N)
$$

and note that $\lim _{N \rightarrow \infty} K(N)=K$. In Fig. 2, we plot $K$, determined from (7), and $K(N)$ for $N \in\{10,20,40,70\}$, determined numerically, versus $\lambda$ for $A_{0}=2, A_{1}=0.1, \sigma_{0}^{2}=$ $3 / 2$, and $\sigma_{1}^{2}=1$ (other parameters are given in the caption). It is seen that the behavior predicted by $K$ holds for $K(N)$. $K(N)$ is seen to be unimodal in $\lambda$ for each $N$. For example, we have that $\lambda^{*}=0.6481 \ldots$, while $K(70)$ is maximized for $\lambda=0.6722 \ldots$. Based on similar simulations over a wide variety of parameters, we conclude that the error exponent accurately predicts features of the error rate even for finite sample sizes.

2) Equispaced Sensors With Failures: Let sensors be equally spaced with common spacing $\bar{d}$ upon deployment, and let $q \in$ $[0,1)$ be the failure rate. Then

$$
f_{d}(x)= \begin{cases}(1-q) q^{n-1}, & x=n \bar{d} \\ 0, & x \neq n \bar{d}\end{cases}
$$

for $n \in\{1,2, \ldots\}$, is the probability density of the spacing between operational sensors. The error exponent is evaluated using (4), (5), and (9). We get

$$
\begin{aligned}
& K=\frac{1}{2}\left\{\ln R-1+\frac{1}{R}\right. \\
& \left.\times\left(1+\frac{\left(e^{A_{0} \bar{d}}-e^{A_{1} \bar{d}}\right)^{2}(1-q)\left(e^{\left(A_{0}+A_{1}\right) \bar{d}}+q\right)}{\left(e^{2 A_{0} \bar{d}}-1\right)\left(q-e^{2 A_{1} \bar{d}}\right)\left(q-e^{\left(A_{0}+A_{1}\right) \bar{d}}\right)}\right)\right\} .
\end{aligned}
$$

The proof follows from algebraic manipulation, including the use of the geometric series, and is omitted for brevity. A general closed form expression for the optimal $\bar{d}$ as a function of $q$ is not available. However, an asymptotic analysis of (10) is given

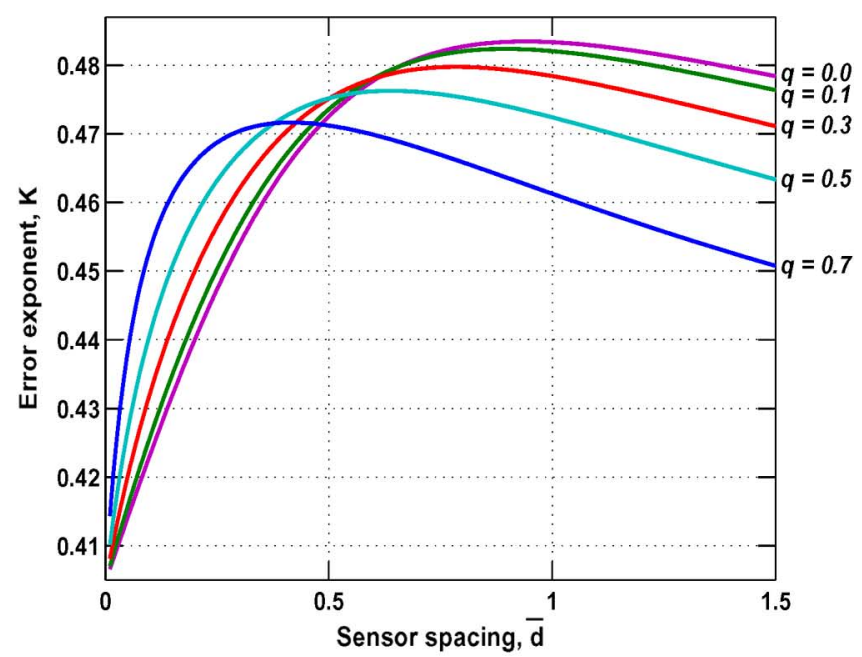

(a)

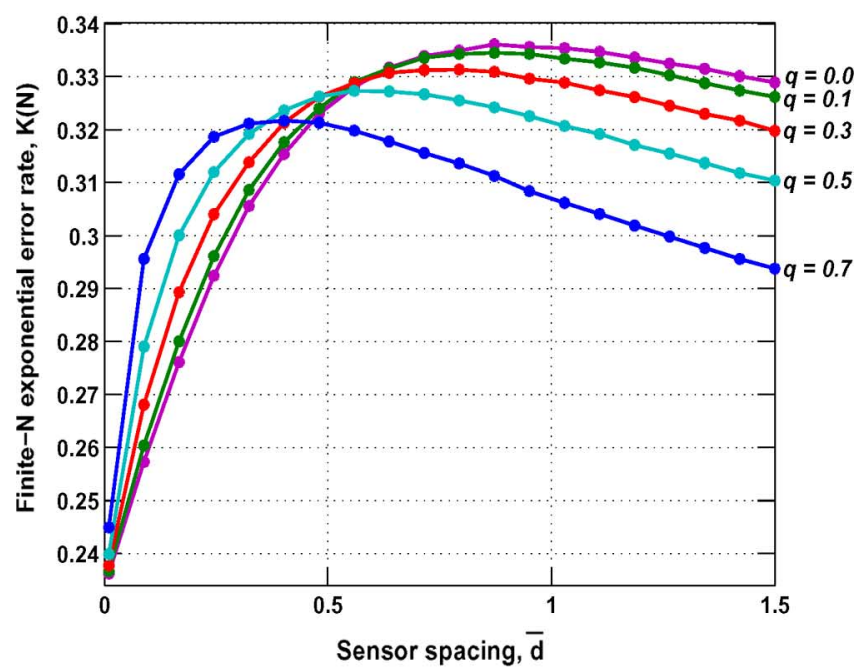

(b)

Fig. 3. NP detection performance versus sensor spacing for geometrically distributed sensors with $q \in\{0.0,0.1,0.3,0.5,0.7\}$ for (a) the error exponent $K$, and (b) the finite- $N$ exponential error rate $K(N)$ with $N=15$. Parameters: $\sigma_{0, I}^{2}=\sigma_{1, I}^{2}=0.5, \sigma_{0}^{2}=1, \sigma_{1}^{2}=5, A_{0}=4$, and $A_{1}=0.1$, and $\alpha=0.01$.

in Appendix III and reveals the following: As $\bar{d} \rightarrow 0, K$ is increasing in both $\bar{d}$ and $q$, and as $\bar{d} \rightarrow \infty, K$ is decreasing in $\bar{d}$ and $q$. Theoretical curves generated from (10) are shown in Fig. 3(a), where we plot $K$ versus $\bar{d}$ for $q \in\{0.0,0.1,0.3,0.5,0.7\}$ (other parameters are given in the caption). It is seen that, for each value of $q$, the error exponent is unimodal in $\bar{d}$ with an optimal $\bar{d}$ that decreases in $q$.

In Fig. 3(b), we plot $K(N)$ when $N=15$ for the same parameters. These numerically determined curves are seen to coincide with theoretical predictions based on $K: K(N)$ is seen to be unimodal in $\bar{d}$ with an optimal spacing that decreases with $q$. When $\bar{d} \ll 1, K(N)$ increases in both $\bar{d}$ and $q$, and when $\bar{d} \gg 1$, $K(N)$ decreases in $\bar{d}$ and $q$. The magnitude of $K$ and $K(N)$ are in relatively close agreement. Based on our simulations across a wide range of parameters, we conclude that $K$ predicts the behavior of $K(N)$ with respect to $\bar{d}$ and $q$. In Fig. 4, we examine the curve corresponding to $q=0.5$ in more detail. We plot $K$ and $K(N)$ for $N \in\{10,20\}$ (other parameters are as before). We see that $K(N)$ increases towards $K$ with increasing $N$. 


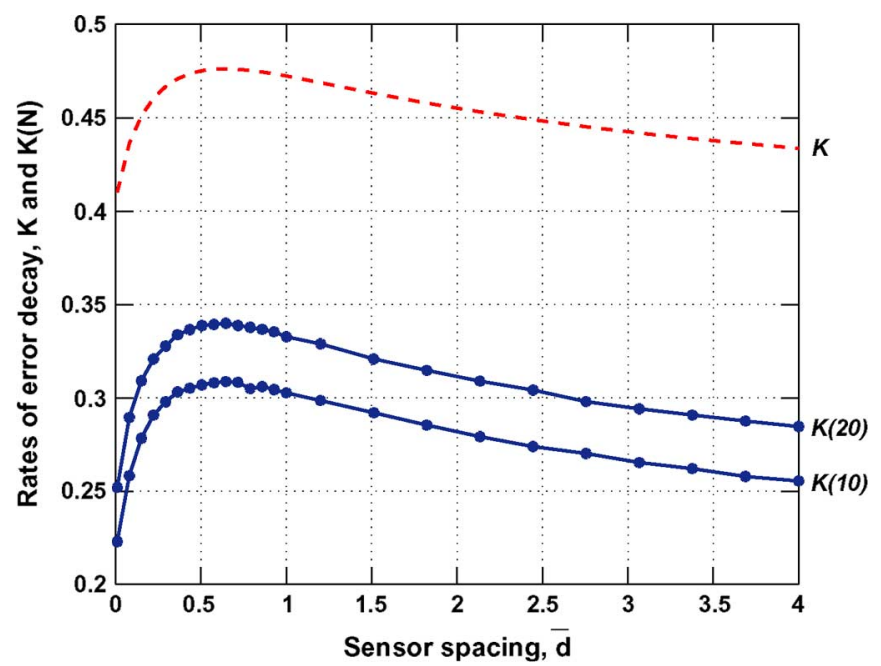

Fig. 4. NP detection performance versus sensor spacing for geometrically distributed sensors with $q=0.5$. We plot $K$ and $K(N)$ for $N \in\{10,20\}$. Parameters: $\sigma_{0, I}^{2}=\sigma_{1, I}^{2}=0.5, \sigma_{0}^{2}=1, \sigma_{1}^{2}=5, A_{0}=4, A_{1}=0.1$, and $\alpha=0.01$.

\section{StATIONARY GAUSS-MARKOV MODEL}

Stationary Gauss-Markov models have also been used to describe communications signals in several related applications (e.g., see [2], [8], and [17]). The model is defined as in the first paragraph of Section II but with two changes. First, we redefine the impulsive noise term to be

$$
z_{j, k} \stackrel{i . i . d .}{\sim} \mathcal{N}\left(0, \sigma_{j}^{2}\left(1-a_{j, k}^{2}\right)\right)
$$

and second, we take the special case that $\sigma_{j, I}^{2}=\sigma_{j}^{2}, j \in\{0,1\}$. Note that $\left\{z_{j, k}\right\}_{k}$ is indeed i.i.d. since $\left\{a_{j, k}\right\}_{k}$ is an i.i.d. sequence. It easy to verify that $\operatorname{VAR}\left(s_{k}^{2}\right)=\sigma_{j}^{2}$, for all $k$, under $\mathcal{H}_{j}$, and that this Gauss-Markov model is stationary. We emphasize that this model is not a special case of the nonstationary model of Section II. For example, (11) introduces dependency of the random quantity $d_{k}$ into $z_{j, k}$.

\section{A. Derivation of the Error Exponent}

We start by evaluating the log likelihood ratio. Following a procedure similar to the nonstationary case, we get

$$
\begin{array}{r}
\ln \frac{p_{0}(\mathbf{s}, \mathbf{d})}{p_{1}(\mathbf{s}, \mathbf{d})}=\frac{1}{2} \ln \frac{\sigma_{1}^{2}}{\sigma_{0}^{2}}+\frac{s_{1}^{2}}{2 \sigma_{1}^{2}}-\frac{s_{1}^{2}}{2 \sigma_{0}^{2}}+\frac{1}{2} \sum_{k=2}^{N} \ln \frac{\sigma_{1}^{2}\left(1-a_{1, k}^{2}\right)}{\sigma_{0}^{2}\left(1-a_{0, k}^{2}\right)} \\
+\frac{1}{2} \sum_{k=2}^{N}\left\{\frac{\left(s_{k}-a_{1, k} s_{k-1}\right)^{2}}{\sigma_{1}^{2}\left(1-a_{1, k}^{2}\right)}-\frac{\left(s_{k}-a_{0, k} s_{k-1}\right)^{2}}{\sigma_{0}^{2}\left(1-a_{0, k}^{2}\right)}\right\}
\end{array}
$$

In Appendix IV, we take the almost sure limit of (12). Doing so, we arrive at the following theorem.

Theorem 3.1: Suppose $\mathbb{E}_{d}\left[\left(1-g_{0}(d)^{2}\right) /\left(1-g_{1}(d)^{2}\right)\right]$, $\mathbb{E}_{d}\left[\left(\left(g_{0}(d)-g_{1}(d)\right)^{4}\right) /\left(1-g_{1}(d)^{2}\right)\right], \mathbb{E}_{d}\left[\left(\left(g_{0}(d)-g_{1}(d)\right)^{2}(1-\right.\right.$ $\left.\left.\left.g_{0}(d)^{2}\right)\right) /\left(\left(1-g_{1}(d)^{2}\right)^{2}\right)\right]$, and $\mathbb{E}_{d}\left[\mid \ln \left(0\left(1-g_{1}(d)^{2}\right) /(1-\right.\right.$ $\left.\left.\left.g_{0}(d)^{2}\right)\right) \mid\right]$ are each bounded from above. Then the NP error exponent for the detection of stationary Gauss-Markov signals with randomly placed sensors is given by

$$
\begin{aligned}
K=\frac{1}{2}\{\ln R & -1+\mathbb{E}_{d}\left[\ln \left(\frac{1-g_{1}(d)^{2}}{1-g_{0}(d)^{2}}\right)\right] \\
& \left.+\frac{1}{R} \mathbb{E}_{d}\left[\frac{1-2 g_{0}(d) g_{1}(d)+g_{1}(d)^{2}}{1-g_{1}(d)^{2}}\right]\right\} .
\end{aligned}
$$

The proof makes use of SLLN-2 (Appendix I). Again, we emphasize that $f_{d}($.$) may be either a continuous or discrete distri-$ bution. In the special case that $d$ is deterministic, (13) matches the NP error exponent that we previously derived in [19], as it must. It is often the case that either $g_{0}(d) \geq g_{1}(d)$ or $g_{1}(d) \geq$ $g_{0}(d), \forall d \in(0, \infty)$, i.e., the signal often decays "uniformly faster" with respect to distance under one of the hypotheses. When either relation holds, the technical conditions required by Theorem 3.1 simplify, as detailed in the following corollary.

Corollary 3.2: Suppose $g_{0}(d) \geq g_{1}(d), \forall d$. Then a necessary and sufficient condition for Theorem 3.1 to hold is $\mathbb{E}_{d}[\ln ((1-$ $\left.\left.\left.g_{1}(d)^{2}\right) /\left(1-g_{0}(d)^{2}\right)\right)\right]<\infty$. Alternatively, suppose $g_{1}(d) \geq$ $g_{0}(d), \forall d$. Then sufficient conditions for Theorem 3.1 to hold are $\mathbb{E}_{d}\left[\left(1-g_{0}(d)^{2}\right) /\left(1-g_{1}(d)^{2}\right)\right]^{2}<\infty$ and $-\mathbb{E}_{d}[\ln ((1-$ $\left.\left.\left.g_{1}(d)^{2}\right) /\left(1-g_{0}(d)^{2}\right)\right)\right]<\infty$.

The proof of the first claim of the corollary follows by upper bounding the argument in each of the first three expectations (in the conditions for Theorem 3.1 to hold) by 1 . The proof of the second claim follows by upper bounding the argument in each of the first three expectations by $\left(\left(1-g_{0}(d)^{2}\right) /\left(1-g_{1}(d)^{2}\right)\right)^{2}$.

\section{B. Example-Exponentially Spaced Sensors With Failures}

We evaluate (13) for exponentially distributed sensors with independent failures. The probability density of the spacing between two consecutive operational sensors is given in (6), and we again use the correlation model (5). In Appendix V, it is shown that the technical conditions of Theorem 3.1 hold, and that substituting (5) and (6) into (13) yields

$$
\begin{gathered}
K=\frac{1}{2}\left\{\ln R-\frac{R+1}{R}+\Psi\left(1+\frac{\lambda_{q}}{2 A_{0}}\right)-\Psi\left(1+\frac{\lambda_{q}}{2 A_{1}}\right)\right. \\
\left.+\frac{\lambda_{q}}{R A_{1}}\left[\Psi\left(\frac{1}{2}+\frac{A_{0}+\lambda_{q}}{2 A_{1}}\right)-\Psi\left(\frac{\lambda_{q}}{2 A_{1}}\right)\right]\right\}
\end{gathered}
$$

where again $\lambda_{q} \triangleq \lambda(1-q)$, and where $\Psi(x)$ is the Psi function [11, p. 943].

When sensors are sparsely placed $(\lambda \ll 1)$, consecutive signal samples approach statistical independence under both hypotheses. Therefore, we expect $K$ to depend only on the signal powers, $\left\{\sigma_{j}^{2}\right\}$. Using the fact that $\Psi(x)=-(1) /(x)+O\left(x^{0}\right)$ $[11$, p. 943], it can be shown that

$$
\lim _{\lambda \rightarrow 0} K=\ln R+\frac{1}{R}-1 \text {. }
$$

When $R=1$, the error exponent is 0 , as expected. When sensors are densely placed $(\lambda \gg 1)$, we use the asymptotic expansion, $\Psi(x) \rightarrow \ln (x)$ for large $x[11$, p. 943$]$ to find that

$$
\lim _{\lambda \rightarrow \infty} K=\ln \left(R \frac{A_{1}}{A_{0}}\right)+\left(R \frac{A_{1}}{A_{0}}\right)^{-1}-1 .
$$




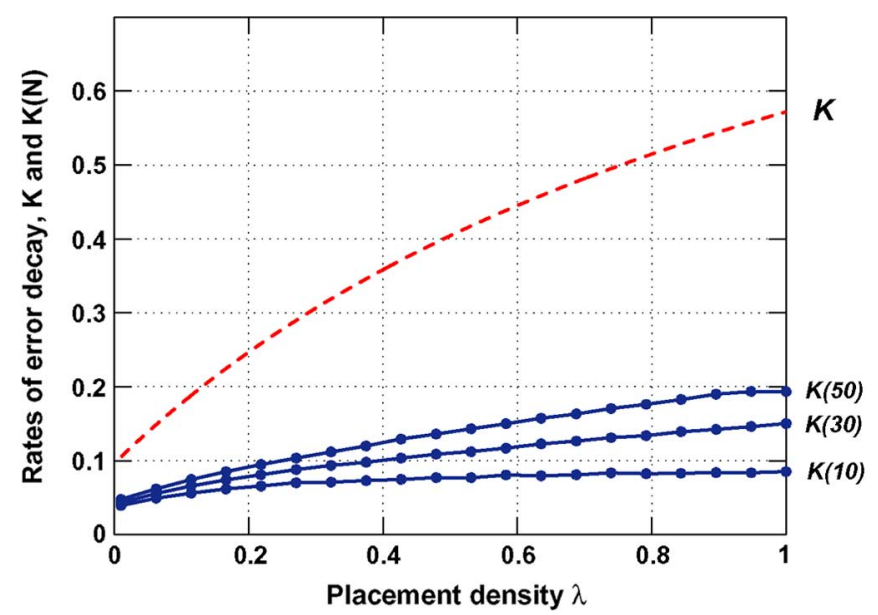

Fig. 5. NP detection performance versus placement density for exponentially distributed sensors. Plot of $K$ and $K(N)$ (for $N \in\{10,30,50\}$, determined numerically) versus $\lambda$ for $A_{0}=2, A_{1}=0.2, \sigma_{0}^{2}=1 / 2, \sigma_{1}^{2}=1, q=0$, and $\alpha=0.01$.

The minimum with respect to $R$ occurs when $R=A_{0} / A_{1}$, for which the error exponent is equals zero. Note that $A_{0} / A_{1}>$ $1(<1)$ implies that the minimum occurs for $R>1(<1)$. This reflects the intuitive fact that detection is harder when the hypothesis with the more strongly correlated signal is also the hypothesis for which the signal variance is greater.

In Fig. 5 we plot $K$ and $K(N)$ (for $N \in\{10,30,50\}$ ) versus $\lambda$ for $A_{0}=2, A_{1}=0.2, \sigma_{0}^{2}=1 / 2$, and $\sigma_{1}^{2}=1$ (other parameters are given in the caption). It is seen that $K(N)$ approaches $K$ as $N$ increases. Next, we determine if the behavior given in (15) and (16) holds for finite $N$ as well. In Fig. 6(a) and (b), we plot $K(N)$ for $N=50$ versus $R$ for progressively smaller values of $\lambda, \lambda \in\{0.2,0.1,0.0001\}$, and progressively larger values of $\lambda, \lambda \in\{2,5,100\}$, when $A_{0} / A_{1}=10$ (other parameters are given in the caption). In the case of small $\lambda$, note that the theory holds. We see that as $\lambda$ decreases, the error exponent decreases, approaching a minimum of zero at $R=1$ as predicted by (15). For large $\lambda$, Fig. 6(b) shows that the error exponent is minimized as $\lambda$ increases, approaching a zero around $R=A_{0} / A_{1}=10$, as predicted by (16). We conclude that the asymptotic analysis given above is useful in predicting trends even for finite $N$.

\section{SUMMARY, DiscuSSION, AND FUTURE WORK}

In this paper, we have derived error exponents for the detection of Gauss-Markov signals with random ( $a d$ hoc) sensor spacing. We assumed that spacings were drawn i.i.d. from an arbitrary distribution function $f_{d}($.$) , and assumed arbitrary cor-$ relation-decay functions $g_{j}(x), j \in\{0,1\}$. We provided exact error exponents for both the nonstationary and stationary cases in (4) and (13), respectively. The error exponents were evaluated using specialized forms of the SLLN [20], [22], and were seen to take on algebraically simple forms involving the parameters of the Markov process and expectations of polynomial functions of $g_{j}(d)$ over $f_{d}($.$) .$

In the nonstationary case, we evaluated the error exponent in closed form for two special cases of $f_{d}($.$) : i) exponentially$ distributed sensors with placement density $\lambda$ and failure rate $q$ (7), and ii) equally spaced sensors with spacing $\bar{d}$ and failure

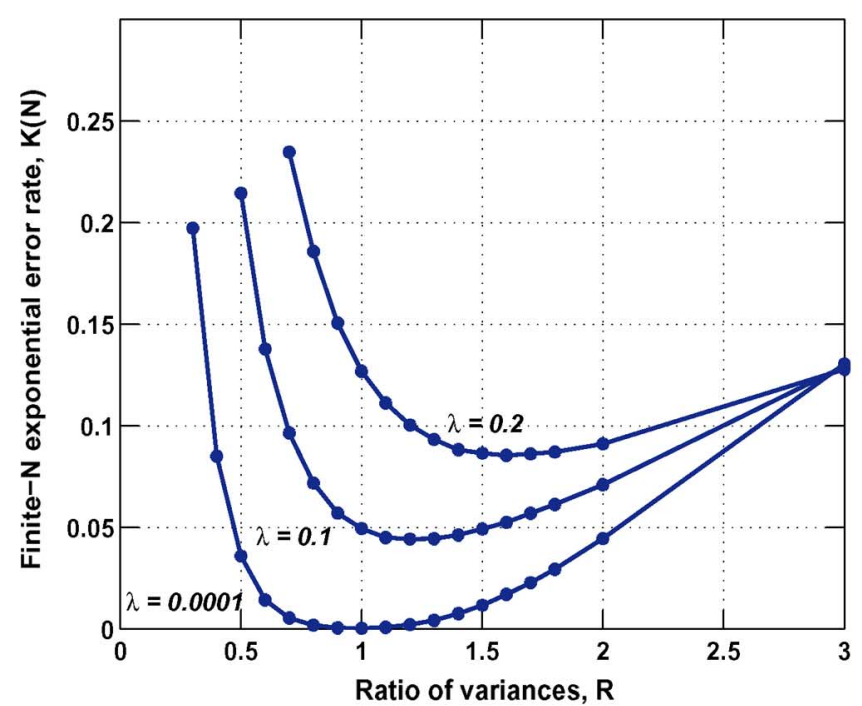

(a)

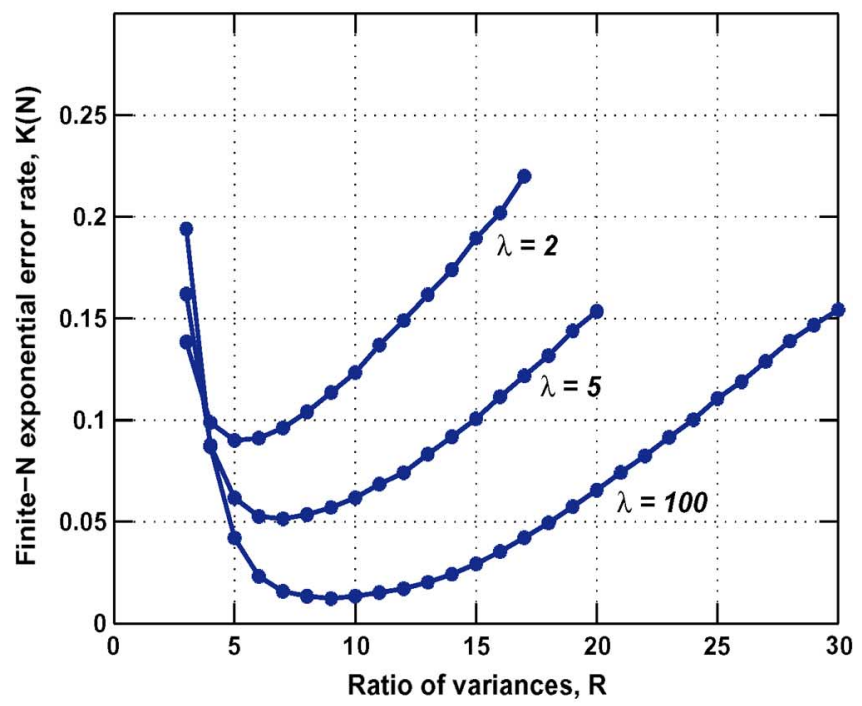

(b)

Fig. 6. NP detection performance versus sensor spacing for exponentially distributed sensors for (a) progressively smaller values of $\lambda$, $\lambda \in\{0.2,0.1,0.0001\}$. (b) Progressively larger values of $\lambda, \lambda \in\{2,5,100\}$. Parameters: $\sigma_{0}^{2}=1, \sigma_{1}^{2}=R, A_{0}=2$, and $A_{1}=0.2, q=0$ and $\alpha=0.01$.

rate $q$ (10). In the first case, the optimal placement density was found in closed form (8). In the second case, properties of the error exponent for small and large density were provable (Section II-B). In the stationary case, we evaluated the error exponent in closed form, in terms of the Psi function, when $f_{d}($.$) corresponds to exponentially distributed sensors with$ failures (14). This expression was seen to simplify in the limit of sparsely and densely placed sensors [(15) and (16), respectively]. Numerical simulations were used throughout to show that the error exponent predicts trends of the simulated error rate accurately for even small data sizes. Thus, the analysis presented here provides many nonobvious and key insights that would not be readily available otherwise.

We discuss assumptions made in this work, and detail avenues of further research. First, we assumed that samples are collected along straight line. If sensors are not located on a straight line, one way to apply the results of this paper is as follows: Generalize 
$g_{j}($.$) to specify the correlation as a function of the Euclidean dis-$ tance separating two consecutively sampled sensors, and let $f_{d}($. be the distribution on this Euclidean distance (this would depend on order in which sensors are sampled, and thus, would be a function of the sampling algorithm). The results (4) and (13) still hold with this new interpretation of $f_{d}($.$) (provided that the signal re-$ tains the Markov property, either directly or for some permutation of the order in which samples are taken), and it would be interesting to see if there exist special cases for which these equations simplify sufficiently to permit a careful analysis. Next, we assumed that sensor observations are free of thermal noise. This assumption is most readily justified when sensors have a high observational SNR. However, the error exponent with noisy observations is clearly of interest. Unfortunately, we are not aware of any techniques which will permit an analysis of (2) in this case. For example, the SLLN techniques in presented in this paper, the spectral factorization method of [24], and the use of certain properties of estimation theory as in [3] all fail to provide ready insights. Finally, we assumed that the distances $\left\{d_{k}\right\}_{k}$ are known or learnt, and that the correlation-decay functions $\left\{g_{j}(x)\right\}_{j}$ are perfectly known. It would be of interest to study the case where $\left\{d_{k}\right\}$ is unknown and/or where $\left\{g_{j}(x)\right\}$ must be estimated.

\section{APPENDIX I}

\section{USEFUl Forms OF THE STRONG LAW OF LARGE NUMBERS}

We reference three forms of the SLLN that will be used in Appendices II and IV. The first version is commonly known and included for completeness.

Theorem SLLN-1 [22, p. 4] (SLLN for i.i.d. Sequences): Let $\left\{X_{i}\right\}_{i \geq 1}$ be an sequence of i.i.d. random variables with $\mathbb{E}\left[X_{1}\right]=$ $\mu$ and $\mathbb{E}\left[\left|X_{1}\right|\right]<\infty$. Then

$$
\lim _{N \rightarrow \infty} \frac{1}{N} \sum_{i=1}^{N} X_{i}=\mu(\text { a.s. }) \text {. }
$$

Theorem SLLN-2 [22, p. 206] (SLLN for Weakly Stationary Sequences): Let $\left\{X_{i}\right\}_{i \geq 1}$ be a weakly stationary sequence of zero-mean random variables, i.e., $\mathbb{E}\left[X_{i}\right]=0$, $\mathbb{E}\left[X_{i}^{2}\right]=\mathbb{E}\left[X_{j}^{2}\right]<\infty$, and $\mathbb{E}\left[X_{i} X_{i+j}\right]=\mathbb{E}\left[X_{1} X_{1+j}\right]$, $\forall i, j \geq 1$. If

$$
\sum_{j=1}^{n}\left|\mathbb{E}\left[X_{1} X_{1+j}\right]\right| \leq D \frac{n}{(\ln n \times \ln (\ln n))^{2}}
$$

for each $n \geq 1$ and some $D<\infty$, then

$$
\lim _{N \rightarrow \infty} \frac{1}{N} \sum_{i=1}^{N} X_{i}=0 \text { (a.s.). }
$$

Theorem SLLN-3 [20] (SLLN for general sequences): Suppose $\left\{X_{i}\right\}_{i \geq 1}$ is a sequence of random variables, not necessarily zero mean, and with arbitrary correlation structure (not necessarily stationary) that is characterized by the existence of a $D<\infty$ and $\beta \in(1, \infty)$ such that

$$
\sum_{t=1}^{N} \sum_{s=1}^{N} \mathbb{E}\left[X_{t} X_{s}\right] \leq D N^{\beta}
$$

for $N \in\{1,2, \ldots\}$. Then for any $\rho>\beta / 2$

$$
\lim _{N \rightarrow \infty} \frac{1}{N^{\rho}} \sum_{t=1}^{N} X_{t}=0 \text { (a.s.). }
$$

\section{APPENDIX II}

PROOF OF (4)

We define the following notation for use in Appendices II and IV: i) If $h(N)$ is a function of $N$, then operator $\lim _{N} h(N)$ is shorthand for $\lim _{N \rightarrow \infty} h(N) / N$ (a.s. in $\mathcal{H}_{0}$ ); ii) define $\bar{a}_{m, n} \triangleq a_{0, m}, \ldots, a_{0, n}$; iii) all expectations are taken under hypothesis $\mathcal{H}_{0}$.

Before proceeding, we will find it useful to state the following facts. Proofs are omitted, as each can be verified through algebraic manipulation. Let $\ell \in\{2,3, \ldots\}$. First, we have that under $\mathcal{H}_{0}$

$$
s_{\ell-1}=\bar{a}_{2, \ell-1} s_{1}+\sum_{k=2}^{\ell-1} z_{0, k} \bar{a}_{k+1, \ell-1}
$$

from which it can be verified that

$$
\mathbb{E}\left[s_{\ell-1}^{2} \mid \mathbf{d}\right]=\bar{a}_{2, \ell-1}^{2} \sigma_{0, I}^{2}+\sigma_{0}^{2} \sum_{m=2}^{\ell-1} \bar{a}_{m+1, \ell-1}^{2},
$$

and

$$
\mathbb{E}\left[s_{\ell-1}^{2}\right]=\mathbb{E}\left[a_{0}^{2}\right]^{\ell-2} \sigma_{0, I}^{2}+\sigma_{0}^{2} \frac{1-\mathbb{E}\left[a_{0}^{2}\right]^{\ell-2}}{1-\mathbb{E}\left[a_{0}^{2}\right]} .
$$

Now, we start from (3) and prove (4). We have

$$
\begin{aligned}
& \lim _{N} \ln \frac{p_{0}(\mathbf{s}, \mathbf{d})}{p_{1}(\mathbf{s}, \mathbf{d})} \\
& \stackrel{(a)}{=} \frac{1}{2} \ln R \\
& +\lim _{N} \frac{1}{2} \sum_{k=2}^{N}\left[\frac{\left(s_{k}-a_{1, k} s_{k-1}\right)^{2}}{\sigma_{1}^{2}}-\frac{\left(s_{k}-a_{0, k} s_{k-1}\right)^{2}}{\sigma_{0}^{2}}\right] \\
& \stackrel{(b)}{=} \frac{1}{2} \ln R+\frac{1}{2} \lim _{N} \sum_{k=2}^{N} \frac{\left(s_{k}-a_{1, k} s_{k-1}\right)^{2}}{\sigma_{1}^{2}} \\
& -\frac{1}{2} \lim _{N} \sum_{k=2}^{N} \frac{z_{0, k}^{2}}{\sigma_{0}^{2}} \\
& \stackrel{(c)}{=} \frac{1}{2}(\ln R-1)+\frac{1}{2 \sigma_{1}^{2}} \lim _{N}\left[\sum_{k=2}^{N}\left(s_{k}-a_{1, k} s_{k-1}\right)^{2}\right] \\
& \stackrel{(d)}{=} \frac{1}{2}(\ln R-1)+\frac{1}{2 \sigma_{1}^{2}} \\
& \times[\underbrace{\lim _{N} \sum_{k=2}^{N} s_{k-1}^{2}\left(a_{0, k}-a_{1, k}\right)^{2}}_{\triangleq F_{1}} \\
& +2 \underbrace{\lim _{N} \sum_{k=2}^{N} z_{0, k} s_{k-1}\left(a_{0, k}-a_{1, k}\right)}_{\triangleq_{F_{2}}}+\underbrace{\lim _{N} \sum_{k=2}^{N} z_{0, k}^{2}}_{\triangleq_{F_{3}}}]
\end{aligned}
$$

where $(a)$ follows since we can ignore deterministic terms that do not scale with $N$ in evaluating the limit, $(b)$ and $(d)$ follow 
from the fact that $s_{k}=a_{0, k} s_{k-1}+z_{0, k}$ under $\mathcal{H}_{0}$, and $(c)$ follows from the observation that $\left(z_{0, k} / \sigma_{0}\right)^{2}$ is an i.i.d. sequence with expected value 1 , and applying the SLLN-1. Next, we evaluate $F_{1}, F_{2}$, and $F_{3}$.

Consider $F_{3}$. Note that $\left\{z_{0, k}^{2}\right\}$ is an i.i.d. sequence with $\mathbb{E}\left[z_{0, k}^{2}\right]=\mathbb{E}\left[\left|z_{0, k}^{2}\right|\right]=\sigma_{0}^{2}<\infty$. Thus, $F_{3}=\sigma_{0}^{2}$ by the SLLN-1.

Consider $F_{2}$. Define $x_{k} \triangleq z_{0, k} s_{k-1}\left(a_{0, k}-a_{1, k}\right)$. We show that SLLN-3 can be applied to $\left\{x_{k}\right\}_{k \geq 2}$. We get

$$
\mathbb{E}\left[x_{k}\right]=\mathbb{E}\left[z_{0, k}\right] \mathbb{E}\left[s_{k-1}\left(a_{0, k}-a_{1, k}\right)\right]=0,
$$

and for $j \in\{1,2, \ldots\}$, that

$$
\begin{aligned}
\mathbb{E}\left[x_{i} x_{i+j}\right]= & \mathbb{E}\left[z_{0, i+j}\right] \\
& \times \mathbb{E}\left[s_{i-1} s_{i+j-1} z_{0, i}\left(a_{0, i}-a_{1, i}\right)\left(a_{0, i+j}-a_{1, i+j}\right)\right] \\
= & 0
\end{aligned}
$$

and

$$
\begin{aligned}
\mathbb{E}\left[x_{\ell}^{2}\right]= & \mathbb{E}\left[z_{0, \ell}^{2}\right] \mathbb{E}\left[\left(a_{0, \ell}-a_{1, \ell}\right)^{2}\right] \mathbb{E}\left[s_{\ell-1}^{2}\right] \\
\stackrel{(a)}{=} & \sigma_{0}^{2} \mathbb{E}\left[\left(a_{0}-a_{1}\right)^{2}\right] \\
& \times\left(\mathbb{E}\left[a_{0}^{2}\right]^{\ell-2} \sigma_{0, I}^{2}+\sigma_{0}^{2} \frac{1-\mathbb{E}\left[a_{0}^{2}\right]^{\ell-2}}{1-\mathbb{E}\left[a_{0}^{2}\right]}\right)
\end{aligned}
$$

where $(a)$ follows from (18). Now, we apply SLLN-3 to $\left\{x_{k}\right\}$. We have

$$
\begin{aligned}
\sum_{\ell=1}^{N} & \sum_{s=1}^{N} \mathbb{E}\left[x_{\ell+1} x_{s+1}\right] \\
= & \sum_{\ell=1}^{N} \mathbb{E}\left[x_{\ell+1}^{2}\right] \\
= & \sigma_{0}^{2} \frac{\mathbb{E}\left[\left(a_{0}-a_{1}\right)^{2}\right]}{1-\mathbb{E}\left[a_{0}^{2}\right]} \\
& \times\left[\sigma_{0, I}^{2}\left(1-\mathbb{E}\left[a_{0}^{2}\right]^{N}\right)-\sigma_{0}^{2}\left(\frac{1-\mathbb{E}\left[a_{0}^{2}\right]^{N}}{1-\mathbb{E}\left[a_{0}^{2}\right]}-N\right)\right] \\
\leq & \sigma_{0}^{2} \frac{\mathbb{E}\left[\left(a_{0}-a_{1}\right)^{2}\right]}{1-\mathbb{E}\left[a_{0}^{2}\right]}\left(\sigma_{0, I}^{2}+\sigma_{0}^{2} N\right) \stackrel{(a)}{\leq} K_{1} N
\end{aligned}
$$

where $K_{1} \in(0, \infty)$ is a constant which can clearly be chosen to satisfy $(a)$ for all $N$ since $\mathbb{E}\left[a_{0}^{2}\right]<1$ by assumption of the theorem. Fixing $\beta \in(1,2)$ and taking $\rho=1$ in the conditions for SLLN-3, we conclude that $F_{2}=0$.

Next, we evaluate $F_{1}$. Define $x_{k} \triangleq\left(a_{0, k}-a_{1, k}\right)^{2} s_{k-1}^{2}$ and $u_{k} \triangleq \mathbb{E}\left[x_{k}\right]=\mathbb{E}\left[\left(a_{0}-a_{1}\right)^{2}\right] \mathbb{E}\left[s_{k-1}^{2}\right]$. We have

$$
\begin{aligned}
F_{1} & =\lim _{N} \sum_{k=2}^{N}\left(x_{k}-u_{k}\right)+\lim _{N} \sum_{k=2}^{N} u_{k} \\
& \stackrel{(a)}{=} \lim _{N} \sum_{k=2}^{N}\left(x_{k}-u_{k}\right)+\sigma_{0}^{2} \frac{\mathbb{E}\left[\left(a_{0}-a_{1}\right)^{2}\right]}{1-\mathbb{E}\left[a_{0}^{2}\right]} \\
& \stackrel{(b)}{=} \sigma_{0}^{2} \frac{\mathbb{E}\left[\left(a_{0}-a_{1}\right)^{2}\right]}{1-\mathbb{E}\left[a_{0}^{2}\right]}
\end{aligned}
$$

where (a) follows from (18), and (b) follows by applying SLLN-3 to the zero-mean sequence $\left\{v_{k}\right\}$ where $v_{k} \triangleq x_{k}-u_{k}$. The proof that the SLLN-3 can indeed be applied to the (nonstationary) sequence $\left\{v_{k}\right\}$ is nontrivial and lengthy. Therefore, we pause momentarily to remark that if we accept (20) without further proof, then by substituting our results for $F_{1}, F_{2}$, and $F_{3}$ into (19), we have arrived at (4), as desired.

Now, we use the remainder of this appendix to show that the SLLN-3 can be applied to $\left\{v_{k}\right\}$. Before proceeding, we state a second set of facts. The first fact is that, for $m>\ell$

$$
\begin{aligned}
& \mathbb{E}\left[s_{m-1}^{2} s_{\ell-1}^{2} \mid \mathbf{d}\right]=3 \bar{a}_{\ell, m-1}^{2} \mathbb{E}\left[s_{\ell-1}^{2} \mid \mathbf{d}\right]^{2} \\
&+\sigma_{0}^{2} \mathbb{E}\left[s_{\ell-1}^{2} \mid \mathbf{d}\right] \sum_{q=\ell}^{m-1} \bar{a}_{q+1, m-1}^{2}
\end{aligned}
$$

This follows from $s_{m-1}=\bar{a}_{\ell, m-1} s_{\ell-1}+$ $\sum_{q=\ell}^{m-1} z_{0, q} \bar{a}_{q+1, m-1}$. The second is that $\forall \ell \geq 2$

$$
\mathbb{E}\left[\mathbb{E}\left[s_{\ell-1}^{2} \mid \mathbf{d}\right]^{2}\right]<C_{0}
$$

for some $C_{0}<\infty$. The proof is lengthy, and thus omitted. However, the technique relies on expanding $\mathbb{E}\left[s_{\ell-1}^{2} \mid \mathbf{d}\right]^{2}$ according to (17) and bounding each term in the expansion. The third fact is that, for $\ell \in\{2,3, \ldots\}$

$$
\mathbb{E}\left[s_{\ell}^{4}\right]<C_{1}
$$

for some $C_{1}<\infty$. To see this, we first establish that $\mathbb{E}\left[s_{\ell}^{4}\right] \leq$ $3 \mathbb{E}\left[\mathbb{E}\left[s_{\ell-1}^{2} \mid \mathbf{d}\right]^{2}\right]$ using the law of iterated expectation and the expression for the fourth moment of a Gaussian random variable, and then apply (22).

We are now ready to show that the SLLN-3 applies to $\left\{v_{k}\right\}$. Define

$$
R_{\ell, m} \triangleq \mathbb{E}\left[v_{\ell} v_{m}\right]=\underbrace{\mathbb{E}\left[x_{\ell} x_{m}\right]}_{\triangleq F_{4}}-\underbrace{u_{\ell} u_{m}}_{\triangleq_{F_{5}}} .
$$

Our approach is to upper bound $R_{\ell, m}$ by lower bounding $F_{5}$ and upper bounding $F_{4}$, and then showing that this upper bound satisfies the condition of SLLN-3. Hence, $R_{\ell, m}$ satisfies SLLN-3.

We lower bound $F_{5}$ for $m>\ell$. We have (24), shown at the bottom of the next page, where $(a)$ follows from substitution of (18), and $(b)$ follows from neglecting the two positive terms in the proceeding equation, and since $\mathbb{E}\left[a_{0}^{2}\right]^{\ell+m-4} \geq 0$.

Similarly, we upper bound $F_{4}$ for $m>\ell$. First, we have

$$
\begin{aligned}
F_{4}= & \mathbb{E}\left[\left(a_{0, \ell}-a_{1, \ell}\right)^{2}\left(a_{0, m}-a_{1, m}\right)^{2} \mathbb{E}\left[s_{\ell-1}^{2} s_{m-1}^{2} \mid \mathbf{d}\right]\right] \\
\stackrel{(a)}{=} & 3 \mathbb{E}\left[a_{0}^{2}\right]^{m-\ell-1} \mathbb{E}\left[\left(a_{0}-a_{1}\right)^{2} a_{0}^{2}\right] \mathbb{E}\left[\left(a_{0}-a_{1}\right)^{2}\right] \\
& \times \mathbb{E}\left[\mathbb{E}\left[s_{\ell-1}^{2} \mid \mathbf{d}\right]^{2}\right]+\sigma_{0}^{2} \mathbb{E}\left[\left(a_{0}-a_{1}\right)^{2}\right]^{2} \frac{1-\mathbb{E}\left[a_{0}^{2}\right]^{m-\ell}}{1-\mathbb{E}\left[a_{0}^{2}\right]} \\
& \times\left(\mathbb{E}\left[a_{0}^{2}\right]^{\ell-2} \sigma_{0, I}^{2}+\sigma_{0}^{2} \frac{1-\mathbb{E}\left[a_{0}^{2}\right]^{\ell-2}}{1-\mathbb{E}\left[a_{0}^{2}\right]}\right)
\end{aligned}
$$


where we have substituted (21) and then (18) in (a). Bounding, we have

$$
\begin{aligned}
F_{4} \stackrel{(a)}{\leq} & K_{1} \mathbb{E}\left[a_{0}^{2}\right]^{m-\ell-1}+\sigma_{0}^{2} \frac{\mathbb{E}\left[\left(a_{0}-a_{1}\right)^{2}\right]^{2}}{1-\mathbb{E}\left[a_{0}^{2}\right]} \\
& \times\left(\mathbb{E}\left[a_{0}^{2}\right]^{\ell-2} \sigma_{0, I}^{2}+\sigma_{0}^{2} \frac{1-\mathbb{E}\left[a_{0}^{2}\right]^{\ell-2}}{1-\mathbb{E}\left[a_{0}^{2}\right]}\right) \\
\leq & K_{1} \mathbb{E}\left[a_{0}^{2}\right]^{m-\ell-1}+K_{2} \mathbb{E}\left[a_{0}^{2}\right]^{\ell-2} \\
& +\sigma_{0}^{4} \frac{\mathbb{E}\left[\left(a_{0}-a_{1}\right)^{2}\right]^{2}}{1-\mathbb{E}\left[a_{0}^{2}\right]}
\end{aligned}
$$

where the constants (with respect to the subscripts $\ell$ and $m$ ) $K_{1}, K_{2} \in(0, \infty)$ clearly exist after using (22) in $(a)$.

Combining (24) and (25), we have

$$
\begin{aligned}
R_{\ell, m}= & F_{4}-F_{5} \\
\leq & K_{1} \mathbb{E}\left[a_{0}^{2}\right]^{m-\ell-1}+K_{2} \mathbb{E}\left[a_{0}^{2}\right]^{\ell-2} \\
& +K_{3}\left(\mathbb{E}\left[a_{0}^{2}\right]^{\ell-2}+\mathbb{E}\left[a_{0}^{2}\right]^{m-2}\right)
\end{aligned}
$$

where $K_{3} \in(0, \infty)$ is a constant w.r.t $\ell$ and $m$ which clearly exists.

Now we apply the SLLN-3 to the sequence $\left\{v_{k}\right\}_{k=2}^{N+1}$. Note

$$
\begin{aligned}
& \sum_{\ell=2}^{N+1} \sum_{m=2}^{N+1} R_{\ell, m} \\
& =2 \sum_{m=2}^{N+1} \sum_{\ell=2}^{m-1} R_{\ell, m}+\sum_{\ell=2}^{N+1} R_{\ell, \ell}^{2} \\
& \quad \stackrel{(a)}{\leq} 2\left[\frac{\left(K_{1}+K_{2}\right)\left(-1+\mathbb{E}\left[a_{0}^{2}\right]^{N}+N\left(1-\mathbb{E}\left[a_{0}^{2}\right]\right)\right)}{\left(1-\mathbb{E}\left[a_{0}^{2}\right]\right)^{2}}\right. \\
& \left.+\frac{K_{3}(N-1)\left(1-\mathbb{E}\left[a_{0}^{2}\right]\right)\left(1-\mathbb{E}\left[a_{0}^{2}\right]^{N}\right)}{\left(1-\mathbb{E}\left[a_{0}^{2}\right]\right)^{2}}\right]+K_{4} N \\
& \leq\left(K_{4}+K_{5}\right) N \quad
\end{aligned}
$$

where $K_{4}$ and $K_{5} \in(0, \infty)$ are constants with respect to $\ell$ and $m$. The term within square brackets in $(a)$ follows from direct substitution of (26), and $K_{4}$ exists as a consequence (23). Thus SLLN-3 with $\beta \in(1,2)$ and $\rho=1$ can be applied to $\left\{v_{k}\right\}$ concluding the proof.

\section{APPENDIX III}

TAYLOR SERIES ANALYSIS OF (10)

From (10), define

$$
Q(\bar{d}) \triangleq \frac{\left(e^{A_{0} \bar{d}}-e^{A_{1} \bar{d}}\right)^{2}(1-q)\left(e^{\left(A_{0}+A_{1}\right) \bar{d}}+q\right)}{\left(e^{2 A_{0} \bar{d}}-1\right)\left(q-e^{2 A_{1} \bar{d}}\right)\left(q-e^{\left(A_{0}+A_{1}\right) \bar{d}}\right)} .
$$

As $\bar{d} \rightarrow 0$, we can ignore the higher-order terms in a Taylor series expansion of $Q(\bar{d})$ about $\bar{d}=0$. We get

$$
Q(\bar{d}) \stackrel{\bar{d} \rightarrow 0}{=} \frac{\left(A_{0}-A_{1}\right)^{2}(1+q)}{2 A_{0}(1-q)} \bar{d}
$$

from which it is clear that $Q(\bar{d})$ is increasing in $\bar{d}$ and $q$.

As $\bar{d} \rightarrow \infty$, we can ignore terms dominated by $e^{C \bar{d}}$, for some $C>0$, in $Q(\bar{d})$. From (27), we get

$$
\begin{aligned}
Q(\bar{d}) \stackrel{\bar{d} \rightarrow \infty}{=}-\frac{\left(e^{A_{0} \bar{d}}-e^{A_{1} \bar{d}}\right)^{2}(-1+q) e^{\left(A_{0}+A_{1}\right) \bar{d}}}{e^{2\left(A_{0}+A_{1}\right) \bar{d}} e^{\left(A_{0}+A_{1}\right) \bar{d}}} \\
=(1-q) \frac{\left(e^{A_{0} \bar{d}}-e^{A_{1} \bar{d}}\right)^{2}}{e^{2\left(A_{0}+A_{1}\right) \bar{d}}} .
\end{aligned}
$$

Clearly, $Q(\bar{d})$ is decreasing in $q$. Taking the derivative with respect to $\bar{d}$, we get

$$
\begin{aligned}
Q^{\prime}(\bar{d})= & -2 \frac{(1-q)}{e^{2\left(A_{0}+A_{1}\right) \bar{d}}}\left(e^{A_{0} \bar{d}}-e^{A_{1} \bar{d}}\right)\left(A_{1} e^{A_{0} \bar{d}}-A_{0} e^{A_{1} \bar{d}}\right) \\
= & -2 \frac{(1-q)}{e^{2\left(A_{0}+A_{1}\right) \bar{d}}}\left(e^{A_{0} \bar{d}}-e^{A_{1} \bar{d}}\right) \\
& \times\left(e^{A_{0} \bar{d}+\ln A_{1}}-e^{A_{1} \bar{d}+\ln A_{0}}\right)
\end{aligned}
$$

$$
\begin{aligned}
& F_{5}= \mu_{\ell} \mu_{m} \\
&= \mathbb{E}\left[\left(a_{0}-a_{1}\right)^{2}\right]^{2} \mathbb{E}\left[s_{\ell-1}^{2}\right] \mathbb{E}\left[s_{m-1}^{2}\right] \\
& \stackrel{(a)}{=} \mathbb{E}\left[\left(a_{0}-a_{1}\right)^{2}\right]^{2} \\
& \times\{\sigma_{0}^{4} \frac{\left(1-\mathbb{E}\left[a_{0}^{2}\right]^{\ell-2}\right)\left(1-\mathbb{E}\left[a_{0}^{2}\right]^{m-2}\right)}{\left(1-\mathbb{E}\left[a_{0}^{2}\right]\right)^{2}}+\underbrace{\sigma_{0}^{2} \sigma_{0, I}^{2}\left[\mathbb{E}\left[a_{0}^{2}\right]^{\ell-2} \frac{1-\mathbb{E}\left[a_{0}^{2}\right]^{m-2}}{1-\mathbb{E}\left[a_{0}^{2}\right]}+\mathbb{E}\left[a_{0}^{2}\right]^{m-2} \frac{1-\mathbb{E}\left[a_{0}^{2}\right]^{\ell-2}}{1-\mathbb{E}\left[a_{0}^{2}\right]}\right]}_{\geq 0} \\
&+\underbrace{\mathbb{E}\left[a_{0}^{2}\right]^{\ell+m-4} \sigma_{0, I}^{4}}_{\geq 0}\} \\
& \stackrel{(b)}{\geq} \sigma_{0}^{4} \frac{\mathbb{E}\left[\left(a_{0}-a_{1}\right)^{2}\right]^{2}}{\left(1-\mathbb{E}\left[a_{0}^{2}\right]\right)^{2}}\left(1-\mathbb{E}\left[a_{0}^{2}\right]^{\ell-2}-\mathbb{E}\left[a_{0}^{2}\right]^{m-2}\right)
\end{aligned}
$$




$$
\begin{aligned}
& \stackrel{\bar{d} \rightarrow \infty}{=}-2 \frac{(1-q)}{e^{2\left(A_{0}+A_{1}\right) \bar{d}}}\left(e^{A_{0} \bar{d}}-e^{A_{1} \bar{d}}\right)\left(e^{A_{0} \bar{d}}-e^{A_{1} \bar{d}}\right) \\
& \quad=-2 \frac{(1-q)}{e^{2\left(A_{0}+A_{1}\right) \bar{d}}}\left(e^{A_{0} \bar{d}}-e^{A_{1} \bar{d}}\right)^{2}
\end{aligned}
$$

which is negative. Thus, $Q(\bar{d})$ is decreasing in $\bar{d}$.

\section{APPENDIX IV \\ PROOF OF (13)}

We use the notation defined in the first paragraph of Appendix II. Starting from (12), we prove (13). From (12), we have

$$
\begin{aligned}
& \lim _{N} \ln \frac{p_{0}(\mathbf{s}, \mathbf{d})}{p_{1}(\mathbf{s}, \mathbf{d})} \\
& \stackrel{(a)}{=} \lim _{N} \frac{1}{2} \sum_{k=2}^{N} \ln \left(R \frac{1-a_{1, k}^{2}}{1-a_{0, k}^{2}}\right) \\
& +\lim _{N} \frac{1}{2} \sum_{k=2}^{N}\left\{\frac{\left(s_{k}-a_{1, k} s_{k-1}\right)^{2}}{\sigma_{1}^{2}\left(1-a_{1, k}^{2}\right)}-\frac{\left(s_{k}-a_{0, k} s_{k-1}\right)^{2}}{\sigma_{0}^{2}\left(1-a_{0, k}^{2}\right)}\right\} \\
& \stackrel{(b)}{=} \frac{1}{2} \ln R+\frac{1}{2} \mathbb{E}\left[\ln \frac{1-a_{1}^{2}}{1-a_{0}^{2}}\right] \\
& +\lim _{N} \frac{1}{2} \sum_{k=2}^{N}\left\{\frac{\left(s_{k}-a_{1, k} s_{k-1}\right)^{2}}{\sigma_{1}^{2}\left(1-a_{1, k}^{2}\right)}-\frac{z_{0, k}^{2}}{\sigma_{0}^{2}\left(1-a_{0, k}^{2}\right)}\right\} \\
& \stackrel{(c)}{=} \frac{1}{2 \sigma_{1}^{2}} \underbrace{\lim _{N} \sum_{k=2}^{N} \frac{\left(a_{0, k}-a_{1, k}\right)^{2} s_{k-1}^{2}}{\left(1-a_{1, k}^{2}\right)}}_{\triangleq_{G_{1}}} \\
& +\frac{1}{2} \underbrace{\lim _{N} \sum_{k=2}^{N} \frac{z_{0, k}^{2}}{\sigma_{1}^{2}\left(1-a_{1, k}^{2}\right)}}_{\triangleq_{G_{2}}} \\
& +\underbrace{\lim _{N} \sum_{k=2}^{N} \frac{z_{0, k}\left(a_{0, k}-a_{1, k}\right) s_{k-1}}{\sigma_{1}^{2}\left(1-a_{1, k}^{2}\right)}}_{\triangleq_{G_{3}}}+\frac{1}{2} \mathbb{E}\left[\ln R \frac{1-a_{1}^{2}}{1-a_{0}^{2}}\right]-\frac{1}{2}
\end{aligned}
$$

where $(a)$ follows since we can ignore deterministic terms that do not scale with $N$ in evaluating the limit, the appearance of $z_{0, k}$ in $(b)$ follows by definition, the expectation in $(b)$ follows from SLLN-1 since $\left\{\ln \left(\left(1-a_{1, k}\right) /\left(1-a_{0, k}\right)\right)\right\}_{k}$ is an i.i.d. sequence and since $\mathbb{E}\left[\left|\ln \left(\left(1-a_{1, k}\right) /\left(1-a_{0, k}\right)\right)\right|\right]<\infty$ by assumption of the theorem, the first three terms in $(c)$ follow from the fact that $s_{k}=a_{0, k} s_{k-1}+z_{0, k}$ under $\mathcal{H}_{0}$, and the last term in $(c)$ from the observation that $\left(z_{0, k}^{2}\right) /\left(\sigma_{0}^{2}\left(1-a_{0, k}^{2}\right)\right)$ is an i.i.d. sequence with expected value 1 .

Next, we evaluate $G_{1}, G_{2}$, and $G_{3}$. To evaluate $G_{2}$, define $x_{k} \triangleq\left(z_{0, k}^{2} /\left(\sigma_{1}^{2}\left(1-a_{1, k}^{2}\right)\right)\right)$ and note that $\left\{x_{k}\right\}$ is an i.i.d. sequence. By the SLLN-1, we get

$$
G_{2}=\mathbb{E}\left[x_{k}\right]=\mathbb{E}\left[\mathbb{E}\left[x_{k} \mid d_{k}\right]\right]=\frac{1}{R} \mathbb{E}\left[\frac{1-a_{0}^{2}}{1-a_{1}^{2}}\right]
$$

provided that $\mathbb{E}\left[\left|x_{k}\right|\right]=\mathbb{E}\left[x_{k}\right]<\infty$, which is true by assumption of the theorem.

To evaluate $G_{3}$, define $x_{k} \triangleq \quad\left(\left(z_{0, k}\left(a_{0, k} \quad-\right.\right.\right.$ $\left.\left.\left.a_{1, k}\right) s_{k-1}\right) /\left(\sigma_{1}^{2}\left(1-a_{1, k}^{2}\right)\right)\right)$. Then note 4

$$
\begin{aligned}
\mathbb{E}\left[x_{k}\right] & =\mathbb{E}\left[\mathbb{E}\left[x_{k} \mid \mathbf{d}\right]\right] \\
& =\mathbb{E}[\underbrace{\mathbb{E}\left[z_{0, k} \mid \mathbf{d}\right]}_{=0} \mathbb{E}\left[\frac{\left(a_{0, k}-a_{1, k}\right) s_{k-1}}{1-a_{1, k}^{2}} \mid \mathbf{d}\right]]=0
\end{aligned}
$$

that

$$
\begin{aligned}
\mathbb{E}\left[x_{k}^{2}\right] & =\mathbb{E}\left[\frac{\left(a_{0, k}-a_{1, k}\right)^{2}}{\sigma_{1}^{4}\left(1-a_{1, k}^{2}\right)^{2}} \mathbb{E}\left[z_{0, k}^{2} \mid \mathbf{d}\right] \mathbb{E}\left[s_{k-1}^{2} \mid \mathbf{d}\right]\right] \\
& =\left(\frac{\sigma_{0}^{2}}{\sigma_{1}^{2}}\right)^{2} \mathbb{E}_{d}\left[\frac{\left(a_{0}-a_{1}\right)^{2}\left(1-a_{0}^{2}\right)}{\left(1-a_{1}^{2}\right)^{2}}\right] \stackrel{(a)}{<} \infty
\end{aligned}
$$

where $(a)$ follows from assumption of the theorem, and that for $j \in\{1,2, \ldots\}$

$$
\mathbb{E}\left[x_{i} x_{i+j}\right]=\mathbb{E}[\underbrace{\mathbb{E}\left[z_{0, i+j} \mid \mathbf{d}\right]}_{=0} \mathbb{E}[(.) \mid \mathbf{d}]]=0
$$

where the argument in $\mathbb{E}[() \mid. \mathbf{d}]$ has been omitted for brevity. Applying the SLLN-2, we find that $G_{3}=0$.

To evaluate $G_{1}$, note that

$$
G_{1}=\mu+\lim _{N} \sum_{k=2}^{N}\left(x_{k}-\mu\right)
$$

with $x_{k} \triangleq\left(\left(\left(a_{0, k}-a_{1, k}\right)^{2} s_{k-1}^{2}\right) /\left(1-a_{1, k}^{2}\right)\right)$ and $\mu \triangleq \mathbb{E}\left[x_{k}\right]=$ $\sigma_{0}^{2} \mathbb{E}\left[\left(\left(a_{0}-a_{1}\right)^{2}\right) /\left(1-a_{1}^{2}\right)\right]$. We will show that the SLLN-2 can be applied to the sequence $\left\{v_{k}\right\}$, where $v_{k} \triangleq x_{k}-\mu$. Note that $\mathbb{E}\left[v_{k}\right]=0$, and

$$
\begin{gathered}
\mathbb{E}\left[x_{k}^{2}\right]=\mathbb{E}\left[\mathbb{E}\left[s_{k-1}^{4} \mid \mathbf{d}\right] \frac{\left(a_{0, k}-a_{1, k}\right)^{4}}{1-a_{1, k}^{2}}\right] \\
\stackrel{(a)}{=} 3 \sigma_{0}^{4} \mathbb{E}\left[\frac{\left(a_{0}-a_{1}\right)^{4}}{1-a_{1}^{2}}\right] \stackrel{(b)}{<} \infty
\end{gathered}
$$

where $(a)$ follows since $s_{k-1} \sim \mathcal{N}\left(0, \sigma_{0}^{2}\right)$ given $\mathbf{d}$, and $(b)$ by assumption of the theorem. Now, note

$$
\begin{aligned}
& \mathbb{E}\left[x_{i} x_{i+j}\right] \\
& =\mathbb{E}[\frac{\left(a_{0, i}-a_{1, i}\right)^{2}}{1-a_{1, i}^{2}} \frac{\left(a_{0, i+j}-a_{1, i+j}\right)^{2}}{1-a_{1, i+j}^{2}} \underbrace{\mathbb{E}\left[s_{i-1}^{2} s_{i+j-1}^{2} \mid \mathbf{d}\right]}_{\triangleq_{Q}}]
\end{aligned}
$$

${ }^{4}$ The law of iterated expectation is necessary since $z_{0, k}$ and $a_{j, k}$ are not independent without conditioning on $d_{k}$. 
for $j \in\{1,2, \ldots\}$. Using the fact that $s_{i+j-1}=\bar{a}_{i, i+j-1} s_{i-1}+$ $\sum_{\ell=i}^{i+j-1} z_{0, \ell} \bar{a}_{\ell+1, i+j-1}$, it can be verified that

$$
Q=3 \sigma_{0}^{4} \bar{a}_{i, i+j-1}^{2}+\sum_{\ell=i}^{i+j-1} \sigma_{0}^{4}\left(1-a_{0, \ell}^{2}\right) \bar{a}_{\ell+1, i+j-1}^{2} .
$$

Substituting $Q$ into (29), using the fact that $\left\{a_{0, k}\right\}_{k}$ is an i.i.d. sequence, and summing over the resulting geometric series, we get

$$
\begin{aligned}
\mathbb{E}\left[x_{i} x_{i+j}\right]= & 3 \sigma_{0}^{4} \mathbb{E}\left[\frac{\left(a_{0}-a_{1}\right)^{2} a_{0}^{2}}{1-a_{1}^{2}}\right] \mathbb{E}\left[\frac{\left(a_{0}-a_{1}\right)^{2}}{1-a_{1}^{2}}\right] \\
& \times \mathbb{E}\left[a_{0}^{2}\right]^{j-1}+\sigma_{0}^{4} \mathbb{E}\left[\frac{\left(a_{0}-a_{1}\right)^{2}}{1-a_{1}^{2}}\right] \\
& \times\left(\mathbb{E}\left[\frac{\left(a_{0}-a_{1}\right)^{2}\left(1-a_{0}^{2}\right)}{1-a_{1}^{2}}\right] \mathbb{E}\left[a_{0}^{2}\right]^{j-1}\right. \\
& \left.+\mathbb{E}\left[\frac{\left(a_{0}-a_{1}\right)^{2}}{1-a_{1}^{2}}\right]\left(1-\mathbb{E}\left[a_{0}^{2}\right]^{j-1}\right)\right) .
\end{aligned}
$$

Finally, we have for $j \in\{1,2, \ldots\}$,

$$
\begin{aligned}
\mathbb{E}\left[v_{i} v_{i+j}\right]= & \mathbb{E}\left[x_{i} x_{i+j}\right]-\mu^{2} \\
= & 2 \sigma_{0}^{4} \mathbb{E}\left[\frac{\left(a_{0}-a_{1}\right)^{2}}{1-a_{1}^{2}}\right] \mathbb{E}\left[a_{0}^{2}\left(a_{0}-a_{1}\right)^{2}\right] \\
& \times \mathbb{E}\left[a_{0}^{2}\right]^{j-1} .
\end{aligned}
$$

Clearly $\mathbb{E}\left[v_{i} v_{i+j}\right]=C \eta^{j-1}$, where $C \in(0, \infty)$ and $\eta \in(0,1)$ are obvious from (30). Using this result in the SLLN-2, we find that $\left\{v_{\ell}\right\}$ obeys the SLLN. Thus, $G_{1}=\mu$. Finally, substituting the results for $G_{1}, G_{2}$, and $G_{3}$ into (28) and simplifying, we get (13), as desired.

\section{APPENDIX V}

\section{EVALUATION OF THE ERROR EXPONENT-FROM (13) TO (14)}

The following three facts will be useful (see [11] and [11, p. 305])

$$
\begin{aligned}
\Psi(x+1) & =\Psi(x)+1 / x, \\
\Psi(x) & =-\gamma-\frac{1}{x}+\sum_{k=1}^{\infty} \frac{z}{k(k+z)},
\end{aligned}
$$

and

$$
\int_{x=0}^{\infty} \frac{e^{p x}-e^{q x}}{e^{r x}-e^{s x}} d x=\frac{1}{r-s}\left[\Psi\left(\frac{r-q}{r-s}\right)-\Psi\left(\frac{r-p}{r-s}\right)\right]
$$

for $r>s, r>p$, and $r>q$. Also, we make use the following: Let $A, B>0$ be constants. Then

$$
\int_{x=0}^{\infty} \ln \left(1-e^{-A x}\right) e^{-B x} d x=-\frac{1}{B}\left[\Psi\left(\frac{B}{A}\right)+\gamma+\frac{A}{B}\right]
$$

A proof follows:

$$
\begin{aligned}
\int_{x=0}^{\infty} \ln \left(1-e^{-A x}\right) e^{-B x} d x & \stackrel{(a)}{=}-\sum_{k=1}^{\infty} \frac{1}{k} \int_{x=0}^{\infty} e^{-(A k+B) x} d x \\
& \stackrel{(b)}{=}-\frac{1}{B} \sum_{k=1}^{\infty} \frac{1}{k} \frac{B / A}{k+B / A} \\
& \stackrel{(c)}{=}-\frac{1}{B}\left[\Psi\left(\frac{B}{A}\right)+\gamma+\frac{A}{B}\right]
\end{aligned}
$$

where $(a)$ follows from a Taylor series expansion of $\ln (1-$ $x$ ) about $x=0,(b)$ from performing the integration, and $(c)$ from (32).

First, assume that the technical conditions of Theorem 3.1 hold. We evaluate the two terms containing expectations in (13). Recall $\lambda_{q} \triangleq \lambda(1-q)$. For the first term, we have

$$
\begin{aligned}
\mathbb{E}_{d}\left[\ln \frac{1-a_{1}^{2}}{1-a_{0}^{2}}\right]= & \mathbb{E}_{d} \ln \left(1-a_{1}^{2}\right)-\mathbb{E}_{d} \ln \left(1-a_{0}^{2}\right) \\
= & \lambda_{q} \int_{x=0}^{\infty} \ln \left(1-e^{-2 A_{1} x}\right) e^{-\lambda_{q} x} d x \\
& -\lambda_{q} \int_{x=0}^{\infty} \ln \left(1-e^{-2 A_{0} x}\right) e^{-\lambda_{q} x} d x \\
& \stackrel{(a)}{=} \Psi\left(1+\frac{\lambda_{q}}{2 A_{0}}\right)-\Psi\left(1+\frac{\lambda_{q}}{2 A_{1}}\right)
\end{aligned}
$$

where (a) follows by applying (34) and then (31).

For the second term, we have

$$
\begin{aligned}
& \mathbb{E}_{d} {\left[\frac{1-2 a_{0} a_{1}+a_{1}^{2}}{1-a_{1}^{2}}\right] } \\
&= \mathbb{E}_{d}\left[\frac{1-2 e^{-\left(A_{0}+A_{1}\right) d}+e^{-2 A_{1} d}}{1-e^{-2 A_{1} d}}\right] \\
& \stackrel{(a)}{=} \lambda_{q}\left[\int_{x=0}^{\infty} \frac{e^{-\lambda_{q} x}-e^{-\left(A_{0}+A_{1}+\lambda_{q}\right) x}}{1-e^{-2 A_{1} x}} d x\right. \\
&\left.\quad+\int_{x=0}^{\infty} \frac{e^{-\left(2 A_{1}+\lambda_{q}\right) x}-e^{-\left(A_{0}+A_{1}+\lambda_{q}\right) x}}{1-e^{-2 A_{1} x}} d x\right] \\
& \stackrel{(b)}{=} \frac{\lambda_{q}}{A_{1}}\left[\Psi\left(\frac{A_{0}+A_{1}+\lambda_{q}}{2 A_{1}}\right)-\Psi\left(\frac{\lambda_{q}}{2 A_{1}}\right)\right]-1
\end{aligned}
$$

where $(a)$ follows from writing the expectation in terms of an integral and decomposing the resultant fraction into two terms, and $(b)$ follows from applying (33) and then (31). Substituting (35) and (36) into (13) and simplifying yields (14).

We now verify that the technical conditions of Theorem 3.1 hold. It is either the case that $A_{1}>A_{0}$, implying from (5) that $g_{0}(d)>g_{1}(d) \forall d$, or that $A_{0}>A_{1}$, implying that $g_{1}(d)>g_{0}(d) \forall d$. Thus, we can use Corollary 3.2 to verify the technical conditions. Suppose that $A_{1}>A_{0}$. That $\mathbb{E}_{d}\left[\ln \left(\left(1-g_{1}(d)^{2}\right) /\left(1-g_{0}(d)^{2}\right)\right)\right]<\infty$ is evident from (35). 
Suppose $A_{0}>A_{1}$. That $-\mathbb{E}_{d}\left[\ln \left(\left(1-g_{1}(d)^{2}\right) /\left(1-g_{0}(d)^{2}\right)\right)\right]<$ $\infty$ is also evident from (35). Additionally, note that

$$
\frac{1-g_{0}(d)^{2}}{1-g_{1}(d)^{2}}=\frac{1-e^{-2 A_{0} d}}{1-e^{-2 A_{1} d}} \leq \frac{A_{0}}{A_{1}}
$$

in this case, which implies

$$
\mathbb{E}_{d}\left[\frac{1-g_{0}(d)^{2}}{1-g_{1}(d)^{2}}\right]^{2} \leq\left(\frac{A_{0}}{A_{1}}\right)^{2}<\infty .
$$

Thus, the technical conditions of Theorem 3.1 hold.

\section{REFERENCES}

[1] V. Aalo and R. Viswanathan, "On distributed detection with correlated sensors: Two examples," IEEE Trans. Aerosp. Electron. Syst., vol. 25, no. 3, pp. 414-421, May 1989.

[2] I. Abou-Faycal, M. Medard, and U. Madhow, "Binary adaptive coded pilot symbol assisted modulation over Rayleigh fading channels without feedback," IEEE Trans. Commun., vol. 53, no. 6, pp. 1036-1046, Jun. 2005.

[3] I. V. Basawa and D. J. Scott, Asymptotic Optimal Inference for NonErgodic Models, ser. Lecture Notes in Statist.. New York: Springer, 1983, vol. 17.

[4] R. S. Blum and S. A. Kassam, "Optimum distributed detection of weak signals in dependent sensors," IEEE Trans. Inf. Theory, vol. 38, no. 3 , pp. 1066-1079, May 1992.

[5] J.-F. Chamberland and V. V. Veeravalli, "Decentralized detection in wireless sensor systems with dependent observations," presented at the 2nd Int. Conf. Computing, Commun. Contrl. Technol., Austin, TX, Aug. 2004.

[6] J.-F. Chamberland and V. V. Veeravalli, "How dense should a sensor network be for detection with correlated observations?," IEEE Trans. Inf. Theory, vol. 52, no. 11, pp. 5099-5106, Nov. 2006.

[7] J.-G. Chen and N. Ansari, "Adaptive fusion of correlated local decisions," IEEE Trans. Syst., Man, Cybern. C, Appl. Rev., vol. 28, no. 2, pp. 276-281, May 1998.

[8] M. Dong, L. Tong, and B. M. Sadler, "Optimal insertion of pilot symbols for transmissions over time-varying flat fading channels," IEEE Trans. Signal Process., vol. 52, no. 5, pp. 1403-1418, May 2004.

[9] E. Drakopoulos and C.-C. Lee, "Optimum multisensor fusion of correlated local decisions," IEEE Trans. Aerosp. Electron. Syst., vol. 27, no. 4, pp. 5-14, Jul. 1991.

[10] W. Feller, An Introduction to Probability Theory and Its Applications, 3rd ed. New York: Wiley, 1968.

[11] I. S. Gradshteyn and I. M. Ryzhik, Table of Integrals, Series, and Products, 4th ed. New York: Academic, 1983.

[12] R. Gray and L. Davisson, An Introduction to Statistical Signal Processing. Cambridge, U.K.: Cambridge, 2004.

[13] Y. Grenier, "Time-dependent ARMA modeling of nonstationary signals," IEEE Trans. Signal Process., vol. 31, no. 4, pp. 899-911, Aug. 1983.

[14] M. Jachan and G. Matz, "Nonstationary vector AR modeling of wireless channels," presented at the 2005 IEEE Signal Processing Advances for Wireless Communications (SPAWC), New York, Jun. 2005.

[15] S. K. Jayaweera, "Sensor system optimization for Bayesian fusion of distributed stochastic signals under resource constraints," presented at the 2006 Int. Conf. on Acoust., Speech, Signal Process. (ICASSP), Toulouse, France, May 2006.

[16] M. Kam, Q. Zhu, and W. S. Gray, "Optimal data fusion of correlated local decisions in multiple sensor detection systems," IEEE Trans. Aerosp. Electron. Syst., vol. 28, no. 3, pp. 916-920, Jul. 1992.

[17] B. Liang and Z. J. Haas, "Predictive distance-based mobility management for multidimensional PCS networks," IEEE/ACM Trans. Netw. vol. 11, no. 5, pp. 718-732, Oct. 2003.
[18] H. Luschgy, A. Rukhin, and I. Vajda, "Adaptive tests for stochastic processes in the ergodic case," Stoch. Process. Appl., pp. 45-59, 1993.

[19] S. Misra and L. Tong, "Error exponents for target-class detection with nuisance parameters," presented at the 2007 Int. Conf. Acoust., Speech, Signal Process. (ICASSP), Honolulu, HI, Apr. 2007.

[20] B. Ninness, "Strong laws of large numbers under weak assumptions with applications," IEEE Trans. Autom. Control, vol. 45, no. 11, pp. 2117-2122, Nov. 2000.

[21] H. V. Poor, An Introduction to Signal Detection and Estimation. New York: Springer-Verlag, 1994.

[22] W. Stout, Almost Sure Convergence. New York: Academic, 1974.

[23] Y. Sung, S. Misra, L. Tong, and A. Ephremides, "Cooperative routing for distributed detection in large sensor networks," IEEE J. Sel. Areas Commun., vol. 25, no. 2, pp. 471-483, Feb. 2007.

[24] Y. Sung, L. Tong, and H. V. Poor, "Neyman-Pearson detection of Gauss-Markov signals in noise: Closed-form error exponent and properties," IEEE Trans. Inf. Theory, vol. 52, no. 4, pp. 1354-1365, Apr. 2006.

[25] Y. Sung, X. Zhang, L. Tong, and H. V. Poor, "Sensor configuration and activation for field detection in large sensor arrays," IEEE Trans. Signal Process., vol. 56, no. 2, pp. 447-463, Feb. 2008.

[26] E. Tuncel, "Extensions of error exponent analysis in hypothesis testing," presented at the 2005 Int. Conf. Inf. Theory (ISIT), Adelaide, Australia, Sep. 2005.

[27] I. Vajda, Theory of Statistical Inference and Information. Dordrecht, The Netherlands: Kluwer Academic, 1989.

[28] P. K. Varshney, Distributed Detection and Data Fusion. New York: Springer-Verlag, 1997

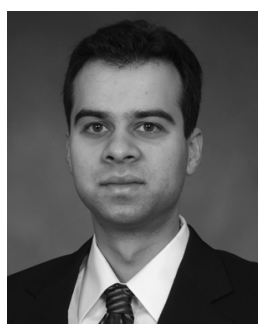

Saswat Misra (M'02) received the B.S. degree in electrical engineering from the University of Maryland, College Park, in 2000 and the M.S. degree in electrical engineering from the University of Illinois at Urbana-Champaign in 2002. He is currently working towards the Ph.D. degree at Cornell University, Ithaca, NY.

He has been a Research Scientist with the Adelphi Laboratory Center of the Army Research Laboratory since $2001 . \mathrm{He}$ is currently studying dynamic spectrum access for cognitive radio systems. He previously studied routing and security in sensor networks, and pilot placement for cellular communications. He has published several technical papers and coinvented two patents (pending) in these areas.

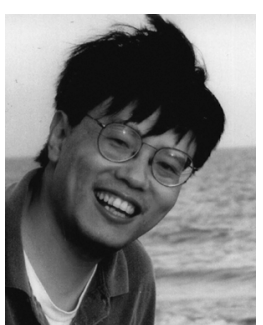

Lang Tong (F'05) received the B.E. degree from Tsinghua University, Beijing, China, in 1985, and the $\mathrm{Ph} . \mathrm{D}$. degree in electrical engineering from the University of Notre Dame, Notre Dame, IN, in 1991.

He joined Cornell University, Ithaca, NY, in 1998, where he is now the Irwin and Joan Jacobs Professor in Engineering. Prior to joining Cornell University, he was with West Virginia University and the University of Connecticut. He was also the 2001 Cor Wit Visiting Professor at the Delft University of Technology, The Netherlands. He was a Postdoctoral Research Affiliate with the Information Systems Laboratory, Stanford University, Stanford, CA, in 1991. He is a coauthor of five student paper awards. His research is in the general area of statistical signal processing, communication systems, and networks.

Dr. Tong received the Outstanding Young Author Award from the IEEE Circuits and Systems Society, the 2004 Best Paper Award (with M. Dong) from the IEEE Signal Processing Society, and the 2004 Leonard G. Abraham Prize Paper Award from the IEEE Communications Society (with P. Venkitasubramaniam and S. Adireddy). He received the Young Investigator Award from the Office of Naval Research. 